\title{
Visualization, Interaction and Simulation Lab at UFRGS
}

\author{
Anderson Maciel, Carla M. Dal Sasso Freitas, Luciana Nedel \\ Universidade Federal do Rio Grande do Sul - Instituto de Informática \\ http://www.inf.ufrgs.br/cg/ \\ \{amaciel, carla, nedel\}@inf.ufrgs.br
}

\begin{abstract}
The Visualization, Interaction and Simulation Laboratory (VISLab) is part of the Computer Graphics, Image Processing and Interaction research group, which started its activities in 1978 developing projects mainly on rendering and animation. Along the years, as new researchers joined the group, new research fields such as image acquisition and analysis, virtual reality, nonconventional interaction, and visualization of complex data started to be investigated. Within this group, VISLab is majorly concerned with research on human-computer interaction, with emphasis on non-conventional, 3D interaction and haptics, and immersive visualization in the context of virtual and augmented reality applications. In this paper, we present the VISLab research focus and the strategy we use to achieve its main goal: to enhance the human with computers, extending the perception capabilities, and improving the human power of action in a natural way. We also briefly describe and illustrate some recent works developed in the lab.
\end{abstract}

Keywords: non-conventional interfaces; virtual and augmented reality; immersive visualization.

\section{Introduction}

In a quest for mechanisms to enhance the human with computers, we have been investigating new technologies and techniques that may contribute to extend the human perception and to enlarge our power of action. This strategy is coupled with the concepts of calm and natural interfaces, where computers become ubiquitous and users live in a fully connected world, being constantly updated through their senses and acting in a precise and natural way. To accomplish our goals, we are conducting research on human computer interaction (HCI) in a broad sense, which includes nonconventional and 3D interaction, haptics, and the use of mobile and wearable devices to allow the implementation of these concepts everywhere. Moreover, our research on HCI is heavily influenced by virtual and augmented reality (VR/AR) setups, which also implies studies on immersive and situated visualization. Results contribute to real applications (e.g., data visualization and health care) and are tested with final users whenever possible.

Our research focus is built on the assumption that human-computer communication should go beyond the eyes to include the touch and the auditory systems. This implies in using the most out of the computational resources available to enhance human activity with all helpful sensory (not only visual) information in harmony with our living environment, while bearing in mind the human tasks the systems are entitled to support. Our goals touch a number of computational and human aspects, which are covered by the projects in the different research lines conducted by the VISLab members.

\section{Recent results}

In this section we provide an overview of some results that illustrate our research focus. The complete list of projects can be found at the lab’s web page.

\section{HCI and 3D interaction}

Earlier, we have proposed a family of 3D interaction techniques based on smartphones for 3D selection and manipulation [1][2]. The use of such devices presents two advantages: they are equipped with a rich set of sensors and fit greatly as pointing devices (1); almost everyone has a smartphone and carries it always and everywhere like an extension of their body.

We have successfully applied smartphone-based 3D interaction techniques for collaborative manipulation of virtual objects in VR and AR. Such novel paradigm for 3D manipulation supports smooth and intuitive collaborative actions. The approach coordinates and combines the multiple users rotations, translations and scales, as well as the camera control [3] (see Fig. 1). It has been proved advantageous for sharing the interaction complexity among many users. When applied in AR [4] this approach combines touch gestures and device movements for fast and precise control of 7DOF transformations. Moreover, the interface creates a shared medium where several users can interact through their point-of-view and simultaneously manipulate 3D virtual augmentations (see Fig. 2).

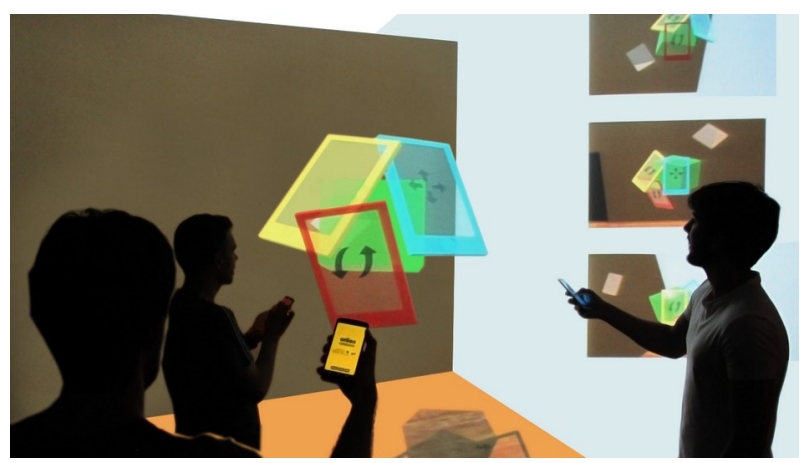

Figure 1. Three users simultaneously driving the object through the virtual environment. The colored rectangles indicate the position and orientation of each user in the VE. The three windows at the right-side show the three personal views. 


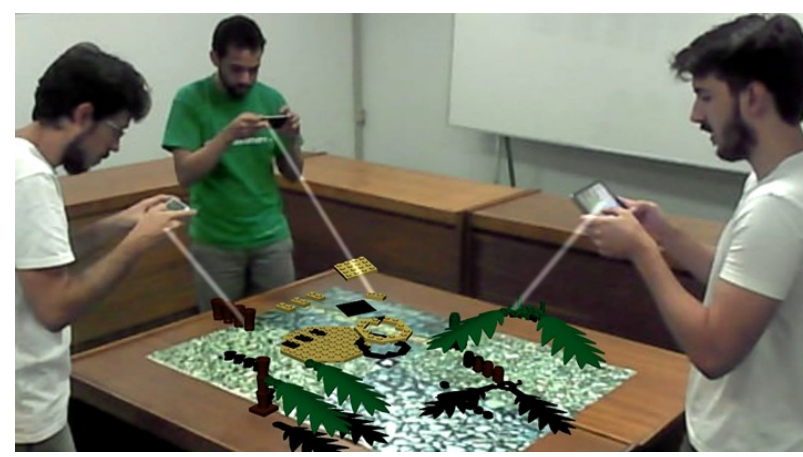

Figure 2. Overview of three persons using our mobile interface to collaboratively manipulate objects in an augmented reality environment.

\section{Haptic interaction}

Touch and force feedback are know to increase immersion and to enrich the sensorial experience in VR. But tactile signals can also be used to communicate ad hoc information in several human-to-human and humancomputer scenarios. While short vibrations that signalize an incoming call or message by a cell phone are ubiquitous, we have worked to extend such simple vibratory notifications so that they are modulated in sets of lexical tactile elements (so called tactons) that put together in sentences compose very expressive languages. One thing we found out is that to increase the language expressiveness without increasing the number of ad hoc tactons, modifier tactile patterns can be used [5]. Perceptual and cognitive factors such as stimuli type and location on the body are some current foci of study.

We explored the actuator density and precision in vibrotactile displays and the acuity for vibration of the head's skin when using such devices [6, 7]. We have also designed and assessed haptic guidance techniques for 3D environments. We designed and assessed a vibrotactile HMD to render the position of objects in a 3D space around the user by varying both stimulus loci around the head and vibration frequency. This combination of stimuli convey respectively direction parallel to the ground and elevation [8].

More recently, we have been investigating the potential uses of other haptic devices like the EXOS Wrist DK2 [9], which is wearable and ungrounded, but still provides rotational stimuli around the wrist. Such stimuli, when combined with the visual information in a virtual environment, can potentially convey a suitable model for balance stimulation and weight perception. The system could have impacting applications for health, with rehabilitation exercises in physiotherapy, to improve or recover one's sense of equilibrium, and to improve general VR metrics such as embodiment and presence.

\section{Immersive analytics and situated visualization}

Current immersive technologies, which combine stereoscopic displays and natural interaction, are being progressively used for information visualization and data analyses. This has been called immersive analytics.
We have investigated the use of an HMD-based environment for the exploration of multidimensional data, represented in 3D scatterplots as a result of dimensionality reduction. We proposed a new modeling for assessment, accounting for the two factors whose interplay determine the impact on the overall task performance: the difference in errors introduced by performing dimensionality reduction to $2 \mathrm{D}$ or $3 \mathrm{D}$, and the difference in human perception errors under different visualization conditions. Instead of the traditional interaction we implement and evaluate an alternative data exploration metaphor where the user remains seated and viewpoint change is only realisable through physical movements. All manipulation is done directly by natural mid-air gestures, with the data being rendered at arm's reach. The virtual reproduction of the analyst's desk aims to increase immersion and enable tangible interaction with controls and two dimensional associated information [10, 11, 12]. More recently, the VirtualDesk approach has been used to evaluate an immersive space-time cube geovisualization for exploring trajectory data [13] (see Fig. 3).
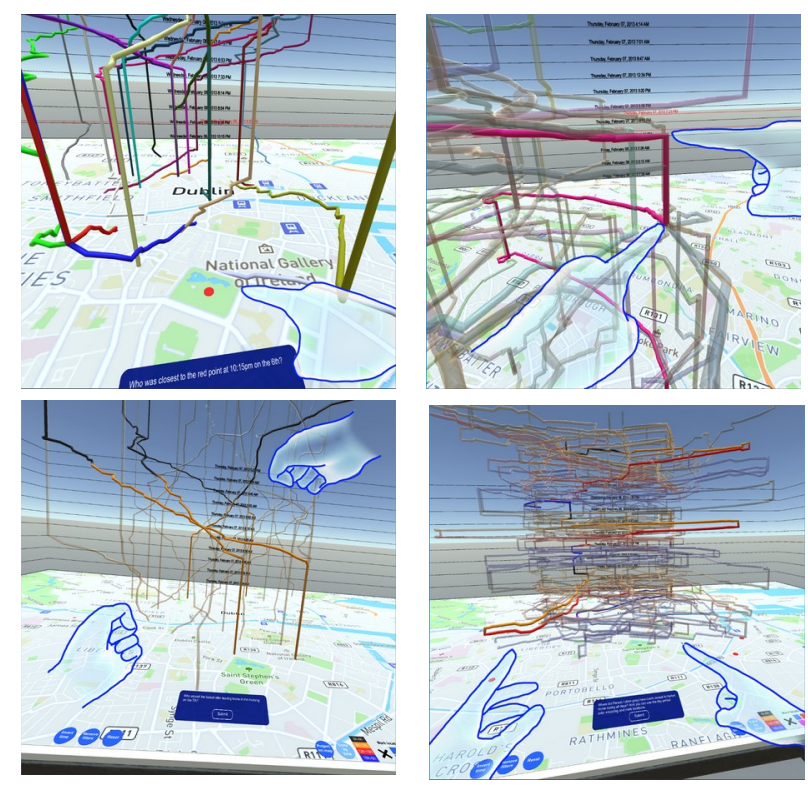

Figure 3. Different tasks being performed in the Immersive condition with Dense data: comparisons of instant distance (top left), stop durations (top right), movement speeds (bottom left) and event locations (bottom right). Blue hand contours added for clarity.

When using augmented reality devices, such as the HoloLens, we refer to such applications as situated visualization or situated analytics. We have also investigated the potential benefits of situated visualization in proof-of-concept applications. For example, an augmented reality user interface was developed to provide information for users to define the most convenient location to sit down in a conference room. This accounts for different sets of arbitrary demands by projecting 3D information directly atop the seats. Qualitative and quantitative data collected from a user study indicated that the augmented reality solution is promising in some senses and may help users to make better decisions [14]. Previously, in another work, we 
used virtual holograms placed on a terrain to guide user navigation instead of the usual heads-up display approach where the augmentations follow the line of sight [15]. More recently, we conducted a study with electromagnetic compatibility (EMC) data, where a user of AR glasses visualize, in a situated fashion, the EM fields around the physical devices that generate them. Situated interaction allows performing data readings accurately and efficiently.

\section{$\underline{\text { Realistic Simulators }}$}

A great motivator for research in VR in the lab is surgery simulation. We have first worked on techniques for modeling organ shapes, joint motion and tissue deformation. Then, we explored collision detection and instrument-tissue interaction, and put that all together in a software framework based on game engines [16]. Currently, the main challenges reside on: creating customized patient models for surgery planning, improving physics-based behavior to simulate real tissue, and proposing new interaction techniques to manipulate medical data.

We have come up with a novel method that uses position-based dynamics for mesh-free cutting simulation. Simulations of cuts on deformable bodies have been an active research subject for more than two decades. However, previous works based on finite elements methods and mass spring meshes cannot scale to complex surgical scenarios. Our solutions include a method to efficiently render force feedback while cutting, an efficient heat diffusion model to simulate electrocautery, and a novel adaptive skinning scheme based on oriented particles [17] (see Fig. 4).

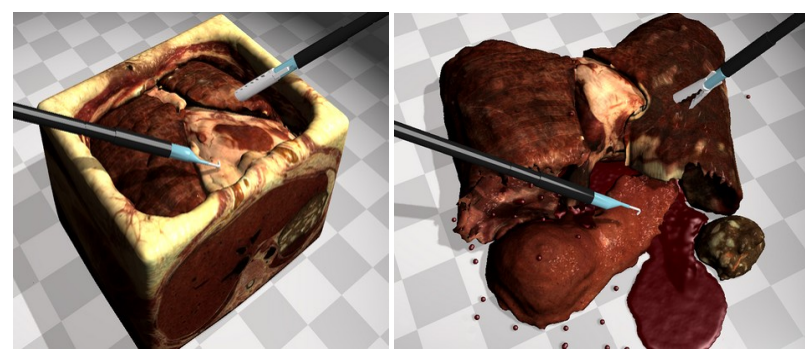

Figure 4. Visual results from a fully dynamic cutting simulation with multiple materials. Thorax organs and other anatomical structures are shown. Nearly 10,000 particles and 30,000 constraints were simulated at haptic framerates.

In another work, we proposed the analysis of videolaparoscopy data to compute the Bidirectional Reflectance Distribution Function (BRDF) of living organs as an input to physically based rendering algorithms, and applied this technique in a case study around the liver with patient-specific rendering under global illumination [18].

Simulating the modeling of virtual dynamic objects in immersive VR is another challenging research topic. While sketching was traditionally a 2D task, and even the new generation of VR devices allowed to sketch in $3 \mathrm{D}$, the drawn models remained essentially static representations. We have developed a new physicsinspired sketching technique built on the top of position- based dynamics to enrich the 3D drawings with dynamic behaviors. A particle-based method allows interacting in real time with a wide range of materials including fluids, rigid bodies, soft bodies and cloths [19] (See Fig. 5).

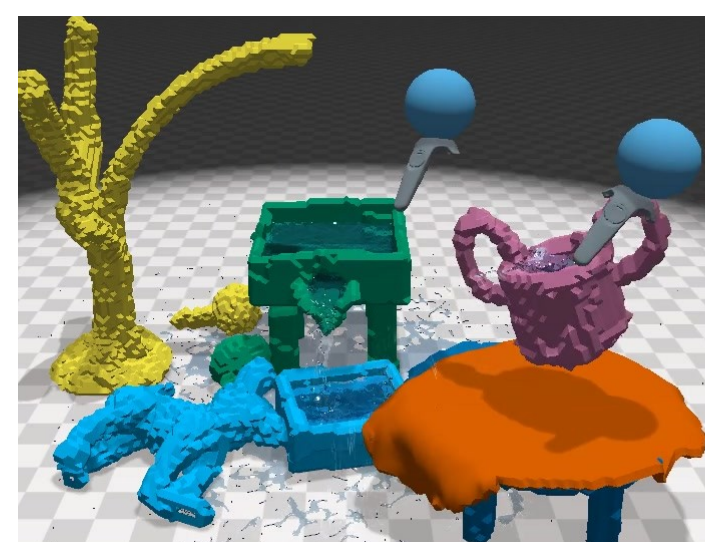

Figure 5. Different dynamic bodies (soft, rigid, fluid) interacting in the same VR scene and with the user controller.

\section{Uniqueness and final comments}

Several factors collaborate to compose our uniqueness in the region and our potential to produce excellent research comparable to top-ranked international institutions. According to the Brazilian Ministry of Education, UFRGS is ranked among the top 5 research universities in Brazil. Its Computer Science (CS) undergraduate program is reputed as number one by the same ministry and recognized by the industry for the excellent education provided. Our Graduate Program in Computer Science is among the best ones and covers most of the CS areas. This entitled us to count on excellent infrastructure and a significant number of undergraduate and graduate research scholarships. Currently, eight faculties and around 40 undergraduate, MSc, and PhD students compose the Graphics, Image Processing and Interaction group. The eight faculties have a varied background for having obtained their $\mathrm{PhD}$ or having spent sabbatical leaves in different, highly qualified institutions worldwide, with which they have maintained cooperation projects with international reference groups over the years. As for VISLab, around 16 students work in our lab on a daily basis, exchanging experiences and taking advantage of the same facilities.

Due to these characteristics, VISLab welcomes students with varied backgrounds but with strong evidence of a research-oriented profile, and also seeks collaboration with other groups to broaden research opportunities.

\section{Acknowledgments}

The authors thank all the students involved in the past and on-going VR projects. Our research has been mainly supported by CNPq, CAPES (Finance Code 001), and FAPERGS. We also acknowledge the financial support from companies in projects over the years (e.g., AESSul, Simedis AG, Microsoft, and Petrobras). 


\section{References}

[1] Debarba, H.; Nedel, L. P.; Maciel, A. (2012) Lop-cursor: Fast and precise interaction with tiled displays using one hand and levels of precision. In: 3DUI. [S.l.]: IEEE, p. 125-132. DOI: 10.1109/3DUI.2012.6184196

[2] Debarba, H. et al. (2013) Disambiguation canvas: A precise selection technique for virtual environments. In: INTERACT 2013. [S.l.]: Springer Berlin Heidelberg, Lecture Notes in Computer Science 8119, p. 388-405. DOI: $10.1007 / 978-3-642-40477-124$

[3] Grandi, J. G. et al. (2017) Design and evaluation of a handheld-based 3d user interface for collaborative object manipulation. In: Proc. 2017 CHI Conference on Human Factors in Computing Systems, p. 5881-5891. DOI: $\underline{10.1145 / 3025453.3025935}$

[4] Grandi, J. G. et al. (2018) Design and assessment of a collaborative $3 \mathrm{D}$ interaction technique for handheld augmented reality. In: Proc. 2018 IEEE Conference on Virtual Reality and 3D User Interfaces, p. 49-56. DOI: 10.1109/VR.2018.8446295

[5] Oliveira, V. J.; Maciel, A. (2014) Introducing the modifier tactile pattern for vibrotactile communication. In: Haptics: Neuroscience, Devices, Modeling, and Applications. Lecture Notes in Computer Science 309-316. DOI: 10.1007/978-3-662-44193-039

[6] Oliveira, V. A. J. et al. (2016) Spatial discrimination of vibrotactile stimuli around the head. In: Proc. 2016 IEEE Haptics Symposium (HAPTICS), p. 1-6. DOI: 10.1109/HAPTICS.2016.7463147

[7] Oliveira, V. A. J. et al. (2016) Localized magnification in vibrotactile hmds for accurate spatial awareness. In: Haptics: Perception, Devices, Control, and Applications, p. 55-64. Cham: Springer International Publishing. DOI: 10.1007/978-3-319-42324-16

[8] Oliveira, V. A. J. et al. (2017) Designing a vibrotactile head-mounted display for spatial awareness in 3d spaces. IEEE Transactions on Visualization and Computer Graphics 23(4): 1409-1417. DOI: 10.1109/TVCG.2017.2657238

[9] Calepso, A. et al. (2020) 3DUI and the phantom limb: Multisensory experience for embodiment of amputation. In: Proc. 2020 IEEE Conference on Virtual Reality and 3D User Interfaces Abstracts and Workshops, p. 517-518. DOI: $10.1109 /$ VRW50115.2020.00110
[10] Wagner Filho, J. A. et al. (2018) Immersive visualization of abstract information: An evaluation on dimensionallyreduced data scatterplots. In: Proc. 2018 IEEE Conference on Virtual Reality and 3D User Interfaces, p. 483-490. DOI: $10.1109 / V R .2018 .8447558$

[11] Wagner Filho, J. A.; Freitas, C.; Nedel, L. (2018) Virtualdesk: A comfortable and efficient immersive information visualization approach. Computer Graphics Forum 37(3): 415-426. DOI: 10.1111/cgf.13430

[12] Wagner Filho, J. A.; Freitas, C. M. D. S.; Nedel, L. (2019) Comfortable immersive analytics with the virtualdesk metaphor. IEEE Computer Graphics and Applications 39(3): 41-53. DOI: 10.1109/MCG.2019.2898856

[13] Wagner Filho, J.; Stuerzlinger, W.; Nedel, L. (2020) Evaluating an immersive space-time cube geovisualization for intuitive trajectory data exploration. IEEE Transactions on Visualization and Computer Graphics 26(1): 514-524. DOI: $10.1109 /$ TVCG.2019.2934415

[14] Guarese, R. et al. (2020) A usability assessment of augmented situated visualization. In: Proc. 2020 IEEE Conference on Virtual Reality and 3D User Interfaces Abstracts and Workshops (VRW), p. 636-637. DOI: 10.1109/VRW50115.2020.00169

[15] Guarese, R. L. M.; Maciel, A. (2019) Development and usability analysis of a mixed reality gps navigation application for the microsoft hololens. In: Advances in Computer Graphics. p. 431-437. Springer International Publishing.

[16] Maciel, A. et al. (2009) Using the physx engine for physics-based virtual surgery with force feedback. The International Journal of Medical Robotics and Computer Assisted Surgery 5: 341-353. DOI: 10.1002/rcs.266

[17] Berndt, I.; Torchelsen, R.; Maciel, A. (2017) Efficient surgical cutting with position-based dynamics. IEEE Computer Graphics and Applications 37(3): 24-31. DOI: 10.1109/MCG.2017.45

[18] Nunes, A. et al. (2017) A laparoscopy-based method for brdf estimation from in vivo human liver. Medical Image Analysis 35: 620-632. DOI: 10.1016/j.media.2016.09.005

[19] Ticona, J. A. et al. (2019) Phys-sketch: Sketching 3D dynamic objects in immersive virtual reality. In: Advances in Computer Graphics, p. 119-130 Springer International Publishing. DOI: $10.1007 / 978-3-030-22514-8 \quad 10$ 